\title{
Anabases
}

ANABASES Traditions et réceptions de l'Antiquité

$23 \mid 2016$

Varia

\section{L'immagine della Grecia in età fascista}

\section{Alessandra Coppola}

\section{OpenEdition}

\section{Journals}

Edizione digitale

URL: http://journals.openedition.org/anabases/5641

DOI: 10.4000/anabases.5641

ISSN: 2256-9421

\section{Editore}

E.R.A.S.M.E.

\section{Edizione cartacea}

Data di pubblicazione: 2 mai 2016

Paginazione: 169-174

ISSN: 1774-4296

\section{Notizia bibliografica digitale}

Alessandra Coppola, "L'immagine della Grecia in età fascista », Anabases [Online], 23 | 2016, Messo

online il 02 juin 2019, consultato il 10 décembre 2020. URL : http://journals.openedition.org/

anabases/5641 ; DOI : https://doi.org/10.4000/anabases.5641 


\title{
L'immagine della Grecia in età fascista
}

\author{
Alessandra Coppola
}

${ }^{66}$ nostri obblighi spirituali verso la Grecia debbono essere intesi con discrezione. Quando Orazio, in un accesso di cavalleria, scrisse i famosi versi sulla Graecia capta che aveva a sua volta conquistato Roma non aveva certo l'intenzione di creare un credito perpetuo a favore dei discendenti degli antichi elleni. Senza contare che questi discendenti hanno, in venti secoli, tanto tralignato dai loro progenitori da non conservare nessun carattere dell'antica divina grecità. La degenerazione bizantina e il dominio turco hanno fabbricato un tipo greco perfettamente balcanizzato".

Questo scriveva nel I940 sul principale quotidiano italiano lo scrittore Curzio Malaparte, ufficialmente inviato ad Atene all'inizio della guerra dell'Italia contro la Grecia ${ }^{1}$. Malaparte non plasmò da sé queste riflessioni, e non fu neppure l'aperta ostilità fra le due nazioni a suggerire in gran fretta questi motivi antichi di rivalità: il repertorio veniva da lontano, e trovava nel conflitto una perfetta applicazione.

In questi anni in cui la crisi economica porta la Grecia alla ribalta, ci si chiede da più parti se sia tollerabile l'affossamento di una nazione europea che vanta un particolare ruolo storico nella formazione della civiltà occidentale. Il riconoscimento di tale ruolo non deriva dalla comprensione o dall'indipendente lettura del passato greco, ma dalla perenne appropriazione che ogni epoca ha creduto di poter realizzare, piegando a sé contenuti e forme. L'eredità culturale greca non venne sempre recepita negli aspetti propri, anzi, la Grecia antica e le sue conquiste

1 Desidero ringraziare Pascal Payen per avermi proposto questa sintesi del mio lavoro Una faccia una razza? Grecia antica e moderna nell'immaginario italiano di età fascista, Roma, Carocci 20I3. "Una faccia una razza" è il motto greco, ancor'oggi citato, che indicava l'affinità fra Italiani e Greci. Per il passo citato vd. C. Malaparte, Gli dei hanno sete, "Corriere della Sera" 3i ottobre I940, p.3. 
intellettuali si prestarono spesso a interpretazioni attualizzanti che adattavano ad altro la sua essenza. L'immagine della Grecia che caratterizza l'età fascista era stata foggiata negli studi ma anche nel panorama politico e risentiva persino delle più recenti acquisizioni della scienza, in un approccio complesso e stratificato, adattabile alle forme di comunicazione e al contesto. Accanto a seri studi si poneva la divulgazione più bieca di messaggi di propaganda o, semplicemente, la ricezione ingenua ma non innocua di temi ufficiali.

L'idea di Grecia della cultura dominante durante il fascismo è il prodotto di un approdo, l'applicazione strumentale di temi e riflessioni che partono dal secolo precedente e che vengono arricchiti di pari passo con il precisarsi dell'ideologia. Già nell'Ottocento un importante momento del recupero delle antiche memorie greche, caricate di messaggi libertari, si ebbe al tempo delle lotte per l'indipendenza, quando il ricordo delle gesta eroiche antiche serviva da stimolo alla resistenza contro il nuovo barbaro e al coinvolgimento europeo. L'Italia ebbe particolarmente a cuore il tema dell'indipendenza greca, in una gradazione di entusiasmi dovuta alla particolare e complessa evoluzione della questione. Fioccarono lavori sulla storia greca moderna, sul popolo e le sue tradizioni, e finché durò l'entusiasmo per le consimili lotte popolari il novello popolo greco era assolutamente visto come l'erede di quello di un tempo; anzi, si contrastavano con vivacità visioni meno esaltanti che derivavano dalla cultura d'oltralpe.

Édmond About pubblicò nel I854, libro sulla Grecia moderna che analizzava pregi e difetti del popolo che tanto stava a cuore all'Europa, senza nascondere critiche ma anche svelando una decisa simpatia. Il libro divenne famoso e fu molto letto, anche in Italia. About prendeva le distanze da un dotto bavarese, Jakob Fallmerayer, che nel I835 aveva invece sollevato seri dubbi sui rapporti tra Greci moderni e Greci antichi, sostenendo che, di fatto, i Greci contemporanei si erano così balcanizzati e slavizzati da essere quasi più albanesi che greci. Queste teorie, divulgate presto in Europa, trovarono detrattori e sostenitori, sia in Grecia che in Italia.

La questione della composizione del popolo greco poteva rimanere un problema storico a sé stante, ma fu presto contaminata da altre idee che caratterizzavano all'epoca la più moderna scienza. Il problema, infatti, scivolò facilmente sul piano biologico dal momento che già a partire dall'età illuministica fino al famoso libro di Nordau (Entartung) la storia dell'essere umano veniva valutata anche in termini di "degenerazione", tanto fisica quanto morale, spirituale, psicologica. Gli studi sull'evoluzione umana, poi, investirono ogni campo, e perfezionarono il tiro. E dall'esame dei cambiamenti alle proposte "eugenetiche" il passo fu breve. Le applicazioni al caso degli ebrei sono tristemente note, ma la teoria degenerativa e le sue conseguenze poterono adattarsi in generale alla storia dei popoli, prima di precisarsi come specifiche misure "sanitarie". Per esempio, in relazione alla Grecia, uno dei fondatori dell'antropologia fisica italiana, Giustiniano Nicolucci, a 
fine Ottocento applicò la pratica della craniologia per verificare le teorie di Fallmerayer e valutare il grado di albanizzazione dei Greci moderni.

Tutto il pensiero evolutivo e degenerativo fu parte integrante dello studio delle razze, e infatti anche il razzismo italiano si confrontò con queste tematiche, e proprio durante il fascismo. Il problema, però, era stabilire quanto dovesse prevalere la biologia sulla storia, o viceversa. E soprattutto quale fosse il ruolo di Roma in tutto questo: notoriamente il problema di Roma, antica e moderna, caratterizzò il dibattito italiano fra Otto e Novecento, esplodendo al tempo della dittatura, quando divenne il tema fondante del regime e un caposaldo dello stato. Roma e l'Italia mussoliniana facevano rivivere i fasti del passato, in perfetta continuità. Dal punto di vista della razza proprio il razzismo "spirituale", quello più attento all'evoluzione storica dei popoli, trovava ampie giustificazioni per collegare le glorie antiche a quelle moderne.

Il problema che presto emerse, nell'esame dell'antica civiltà romana rinata nel presente fascista, era proprio il rapporto con la Grecia, sia a livello storiografico serio sia, di conseguenza, a livello politico. Secondo l'idea prevalente, Roma aveva superato l'Ellade nella realizzazione di un'unità statale solida, aliena dai particolarismi e capace di grande organizzazione. Se in generale si lodavano le acquisizioni genericamente culturali dei Greci, se ne indicava sempre la limitatezza politica. Il primo confronto possibile era naturalmente con la Macedonia, a partire da Filippo fino ad Alessandro. Su questo si sviluppò anche in Italia un dibattito che era partito a suo tempo con le riflessioni di Droysen e i confronti più o meno velati con il suo presente. Attraverso la Macedonia, si valutava l'importanza dell'unificazione contro il particolarismo, vedendo così contrapposte diverse gradazioni di adesione al primato di Roma e del cristianesimo, superiori perché universalistici.

Nei contributi più allineati e nella divulgazione culturale ufficiale, la supremazia di Roma, mai dimenticata, andava affermata sempre più a discapito della Grecia antica, perché in maniera subdola o palese si insinuava il confronto fra Roma mussoliniana e la Grecia attuale. Bisognava quindi calcare i toni contro chi aveva accusato Roma di aver fatto decadere la cultura greca, o evidenziare lo "sfacelo morale" della Grecia antica, pericoloso per la stessa virtù di Roma, per concludere sempre in lode alla Romanità imperiale "che, dopo tante vicende millenarie, rivive in pieno e trionfa oggi" ${ }^{2}$. L'antichità era progressivamente piegata all'ideologia dominante, tanto da diventare simbolo anche della disciplina dell'obbedienza teorizzata dall'idea di "mistica fascista", che prevedeva lo slancio irrazionale e totalizzante verso la persona e il pensiero del duce. Ecco perché si poteva scrivere con

2 G. Cardinali, "La funzione dell'impero romano nell'antichità", in La missione dell'impero di Roma nella storia della civiltà, Atti del V congresso nazionale di studi romani, Roma I938, p. I-I4. 
orgoglio che i Romani erano antirazionalisti e anti-intellettuali e consideravano la cultura greca "oggetto estraneo ai loro doveri di cittadino"3.

A creare un pericoloso miscuglio furono gli approcci di tipo medico-biologico ed evolutivo, che venivano da più lontano. Alessandro, per esempio fu presto oggetto di studio. Cesare Lombroso, negli Studi sul genio aveva ricavato dati sulla "follia morale di Alessandro: accessi di megalomania, di impulsività, di deliri religiosi, non che certe bizzarrie simili a quelle or notate in Napoleone, la gamomania, per esempio... soprattutto spiccata ebbe l'eredità morbosa nella madre dissoluta, invidiosa, egoista, superstiziosa, altera, impetuosa, e nel fratello Filippo Arrideo, parzialmente imbecille". Nonostante ciò, qualche anno più tardi il prof. Bertolotti lo considerava "stupendo campione umano, figura eugenica... senza tare e senza difetti costituzionali, all'infuori di una emiatrofia facciale, dovuta forse a un'azione traumatica durante il parto. Scartata l'eventualità di un torcicollo congenito, resterebbe a discutere quella di un torcicollo patologico post-traumatico, cioè di una lesione ossea atlo-occipitale ${ }^{4 "}$.

Questo tipo di approccio non tardò a sfociare in vera analisi di tipo razziale: per esempio i Cartaginesi, i principali avversari dei Romani, erano attaccati soprattutto in quanto semiti. E gli stessi Greci, sulla scia di precedenti analisi di tipo storico, furono bollati sempre più come degeneri e totalmente estranei ai loro grandi antenati in termini razziali, mentre ci si affannava a dimostrare che invece in Italia la stirpe si era mantenuta intatta nei secoli.

Così stabilita la superiorità romana in termini storici e biologici, era preparato il terreno per lo scontro contemporaneo fra le civiltà, a seguito della dichiarazione di guerra dell'Italia alla Grecia, il 28 ottobre i940. A questo scopo serviva aggiungere la denigrazione della civiltà bizantina, non certo nuova ma ora strumentale, anche in quanto simbolo di un oriente ortodosso che si avvertiva come pericoloso.

In tutto ciò si impegnavano gli intellettuali di regime e naturalmente la stampa più divulgativa, producendo un'ampia ricezione di questo atteggiamento culturale. E alla frequente domanda se i Greci moderni fossero o no i discendenti di quelli di un tempo, così si scriveva in una nota rivista di viaggi, a proposito della conquista romana della Grecia: "Allora, gli antichi Elleni di razza ariana, la cui splendida stirpe si era già affermata non solo nella Grecia propriamente detta ma anche nella Micrasia e nella Magna Grecia, cioè nell'Italia meridionale e nella Sicilia, erano già in gran parte scomparsi, sostituiti rapidamente da elementi semitizzati venuti dall'oriente, da una popolazione, quindi, la quale risulta oggi composta, in complesso, da una secolare mescolanza di Slavi, di Illiri, di Semiti ed altri orientali... Poco o nulla è

3 E. Ciaceri, Da Catone a Cicerone e la Livio a Tacito, "Dottrina fascista" 4, maggio I940, p. $727-733$.

4 M. Bertolotti, La critica medica nella storia. Alessandro Magno, Torino I932, p. I4I. 
rimasto degli Ateniesi e degli Spartani dei tempi di Pericle. Scarse le somiglianze somatiche e spirituali, ma forse piuttosto una semplice coincidenza geografica

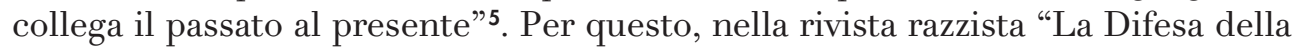
razza" del dicembre I940 si affiancavano visivamente immagini di bellezze classiche ad altre di Greci moderni non proprio avvenenti, ad esaltarne la distanza. In climax discendente, il greco levantino poteva infine diventare semita.

E per contrastare chi accusava l'Italia di attaccare un paese che fu culla di civiltà, si rispondeva che "non contro la Grecia classica, dunque, della quale, in linea ideale, voglio dire di genio, noi Italiani siamo i soli legittimi discendenti, ma contro la Grecia moderna, equivoco stato asservito agli interessi dei plutocrati inglesi, ebrei ed americani, noi siamo scesi in guerra... E insieme alle nostre colonne vittoriose entrerà in Grecia il soffio di quella civiltà, unica al mondo, che prima fiorì sulle sue sponde per poi emigrare definitivamente verso i lidi fatali di Roma". Infatti, "l'amicizia di molti Greci per l'Inghilterra deriva da un'inferiorità razziale acquisita di seguito all'imbastardimento ed ai miscugli con altre razze non esclusa la semitica, per cui il bisogno di servire il padrone si scambia... per affinità elettiva"6.

Certo questi sono gli strilli della propaganda di guerra, ma la base da cui scaturiscono era permeata in ampi strati culturali. Questo si evince anche nella produzione delle memorie di viaggio, dove si registrano impressioni di viaggiatori per lo più già condizionati in partenza. La ricerca del classico non sempre supera la delusione, e in questi casi essa si esprime rielaborando il repertorio ufficiale dell'inferiorità greca e della distanza dei Greci moderni da quelli antichi.

Un esempio viene da un noto docente e critico, Mario Praz, che si recò in Grecia nel ig3r: "Grecia antica e Grecia moderna, cose che si possono tenere distinte solo se sbarchi da un yacht sul luogo di scavo, e ti armi di parocchi, e corri ai ruderi e, un occhio sul libro, l'altro sulla rovina descritta, fai in te ribollire tutti i sedimenti di cultura classica, tutte le ideologie più o meno approssimative di cui si è venuta nutrendo la tua coscienza d'europeo... dopo un viaggio in Grecia la Laus Vitae ti vien voglia di tirarla contro il muro"7. La Laus Vitae è notoriamente uno dei primi canti di Maia, l'opera di D’Annunzio incentrata sulla Grecia. Praz è sconvolto dalla miseria della Grecia contemporanea: "Il supino abbrutimento che scopro su tanti volti di Greci sarebbe l’ultima parola di una razza". E quindi perché darsi da fare

5 I. Bianchi, Le aquile romane tornano in Grecia, in "Le vie d'Italia", dicembre I940, p. I408-I 422.

6 G. Lupi,La tragedia della razza greca, "La Difesa della Razza", 20 gennaio I939, p.33-34.

7 M. Praz, Viaggio in Grecia, a cura di M.Staglieno (s. 1. ma casa ed. Shakespeare and Kafka) I99I, p. II-I5. Il testo fu pubblicato inizialmente sull' "Ambrosiano" dall'aprile al giugno I93I, e poi come libro nel 1942 (Viaggio in Grecia: diario del Ig3I, Roma, Ed. di lettere e viaggi). 
con gli scavi archeologici?: "Se c’è bisogno di missioni in Grecia, c'è prima di tutto bisogno di missioni per demolire tutto ciò che s'è mal costruito nell'età moderna, e costruire e risanare... Il pittoresco greco è già scomparso da un pezzo... è rimasto il detrito, la feccia di un mondo che non è più né moderno né antico, ma è nowhere, nel triste limbo di ciò che non ha ragione d'esistere". La Grecia, dunque, andava per lui "risanata", secondo la terminologia corrente.

Anche uno scrittore di livello, come Alberto Moravia, direttosi in Grecia nel I938, dimostra come si potesse assorbire facilmente la cultura dominante: "Troppo spesso chi si reca in Grecia si illude di ritrovare non troppo degeneri gli ultimi discendenti dei Greci antichi", dove la parola "degeneri" riassume molti anni di riflessioni sullo sviluppo umano e trova applicazione nel popolo che si contrapponeva a Roma e che già nel secolo precedente era stato così svilito nella cultura europea: neppure Byron si era sottratto, ma in tempi più recenti questa denigrazione si era decisamente arricchita dell' ideologia dominante: "La disinvoltura con la quale Roma fece uso della forza per tagliare il nodo gordiano greco non attesta tanto una brutalità d'altronde necessaria, quanto la libertà di spirito di una concezione politica superiore. I Romani avevano il vantaggio di guardare all'avvenire e non al passato ${ }^{8}$."

La guerra, che portò al culmine queste analisi ideologizzate, ebbe come unico pregio, alla fine, quello di spazzare in un sol colpo la totalità di questo tipo di approccio.

\author{
Alessandra Coppola \\ Università degli Studi di Padova \\ Dipartimento dei Beni culturali \\ Piazza Capitaniato, 7 , \\ 35139 - Padova, Italia \\ alessandra.coppola@unipd.it
}

8 Alberto Moravia. Viaggi. Articoli 1930-ıggo, a cura e con introduzione di E. Siciliano, postfazione di T. Tornitore, Milano 1997², p.387-457. 